\title{
LOS SONETOS Y CANCIONES DEL POETA FRANCISCO PETRARCHA DE ENRIQUE GARCÉS. NOTAS SOBRE EL CANZONIERE DE FRANCESCO PETRARCA EN LA AMÉRICA DEL S. XVI ${ }^{1}$
}

\author{
M. ${ }^{a}$ José BerTOMEU MASiÁ \\ Universidad de Valencia
}

\section{RESUMEN}

En la segunda mitad del siglo XVI se realizaron tres traducciones al castellano del Canzoniere de Francesco Petrarca; la única completa es la que publicó en 1591 con el título Los sonetos y canciones del poeta Francisco Petrarcha que traduzia Henrique Garcés de lengua thoscana en castellana, un minero, librero y poeta de origen portugués llamado Enrique Garcés que desarrolló su labor cultural en Perú y mantuvo contacto con los círculos intelectuales más importantes de la segunda mitad del siglo en Hispanoamérica.

Este artículo, tras una breve introducción a la figura de Enrique Garcés y su papel en el Perú virreinal, se centra en el análisis de la traducción para intentar averiguar cuál podría ser el texto italiano del que tradujo Garcés y, por último, estudia la Canción al Pirú a imitación de «Italia mia, ben che'l parlar sia in darno».

Palabras clave: Petrarca, Canzoniere, Enrique Garcés, traducción, Petrarquismo en América.

\begin{abstract}
In the second half of the $16^{\text {th }}$ century three translations to Castilian of were made; only the complete one is the one that was published in 1591: Los sonetos y canciones del poeta Francisco Petrarcha que traduzia Henrique Garcés de lengua thoscana en castellana by a miner, bookseller and poet of Portuguese origin named Enrique Garces who developed his cultural work in Peru and maintained contact with the most important intellectual circles of second half of the century in Hispano-America.

This article, after a brief introduction that looks at the figure of Enrique Garcés and his role in viceroyal Peru, it is centered in the analysis of the translation in order to find out the text that worked Garcés with and, finally, it studies the poem Canción al Pirú a imitación de «Italia mia, ben che'1 parlar sia in darno».
\end{abstract}

Key words: Petrarch, Canzoniere, Translation, Enrique Garcés, Petrarchism in America.

\footnotetext{
${ }^{1}$ La redacción de este artículo ha sido posible gracias a la Beca Predoctoral de Formación de Personal Investigador concedida por la Generalitat Valenciana (Conselleria de Investigació, Universitat i Ciència, Direcció General d'Universitats i Investigació) en 2004 con la referencia CTBPRB/2004/199.
} 
En 1591 se imprimió una traducción del Cancionero de Francesco Petrarca realizada por un polifacético hombre del siglo XVI llamado Enrique Garcés. La obra se titula Los sonetos y canciones del poeta Francisco Petrarcha que traduzía Henrique Garcés de lengua thoscana en castellana ${ }^{2}$, impresa en Madrid en 1591 por el impresor Guillermo Droy.

Se trata de un texto de enorme importancia para la historia de la literatura y sobre todo para la historia de la difusión del petrarquismo en América Latina, pues fue la primera traducción del poeta italiano en dicho continente. De hecho, es la única traducción completa del texto al castellano realizada en la península o fuera de ella, hasta el siglo XX.

Enrique Garcés es un personaje fundamental para conocer cómo se difundió la obra de Petrarca entre los intelectuales y poetas americanos por su labor como traductor pero también por su oficio de librero y promotor de la vida cultural en uno de los grandes centros urbanos de la América del siglo XVI: el Virreinato de Perú. Perú fue el primer centro cultural del continente americano, debido a que fue el primer lugar del mismo en el que se estableció la imprenta, en 1576.

Muchas dudas existen aún entorno a la persona de Enrique Garcés. Sabemos cierto que nació en Oporto, pero la fecha no pone de acuerdo a los especialistas, quienes debaten entre 1520 y 1540. Algunos estudiosos, como Antonio Prieto ${ }^{3}$ o Justo García Morales ${ }^{4}$, afirman que nació entre 1530 y 1540; sin embargo, estas fechas no parecen muy probables pues sabemos seguro que en 1547 estaba ya trabajando en Huancavelica, por lo que debió nacer, como mucho, antes de 1530; en este sentido se pronuncian precisamente Alicia de Colombí ${ }^{5}$ o Estuardo Núñez ${ }^{6}$, quienes se decantan porque el nacimiento de Garcés hubiera tenido lugar entre 1520 y 1525.

Según Estuardo Núñez, siguió estudios de humanidades y de minería en España, cerca de Ciudad Real, antes de embarcarse — cuando tenía alrededor de 25 años - en Sevilla hacia Cartagena, donde llegaría tres meses después para trasladarse a Panamá, y después a Lima. En realidad debió embarcarse a una edad un poco más temprana si, como afirma Justo García Morales, trabajó en Huancavelica «puesto que encontramos su firma estam-

2 Esta será la edición que manejaremos de aquí en adelante en las citas sino indicamos lo contrario. El ejemplar que se encuentra en la Biblioteca de la Universidad de Valencia tiene la signatura BH Z-13/242.

${ }^{3}$ PRIETO, Antonio, Francesco Petrarca. Cancionero, Barcelona, Planeta, 1985, p. CV.

${ }^{4}$ García Morales, Justo, Rimas en vida y muerte de Laura; Triunfos, Madrid, Aguilar, 1963, p. 70.

${ }_{5}$ De Colombí, Alicia, Petrarquismo peruano: Diego Dávalos y Figueroa y la poesía de la Miscelánea Austral, Londres, Tamesis Books Limited, 1985, p. 34, n. 12.

${ }^{6}$ NúŃEZ, Estuardo, «Henrique Garcés, múltiple hombre del Renacimiento», La tradición clásica en el Perú virreinal, Teodoro Hampe, Lima, Sociedad Peruana de Estudios Clásicos: Universidad Nacional Mayor de San Marcos, 1999, p. 129. 
pada en 1547 en dos cartas y un memorial en los que se protesta ante el monarca de la mala política minera seguida por el virrey» ${ }^{7}$.

El hecho cierto es que a mediados de siglo estaba ya en Perú, donde estableció un negocio de librería y papelería de importación, labor que aplazó para comenzar a trabajar en la explotación minera. Su trabajo como minero le granjeó el reconocimiento de las autoridades, debido a su descubrimiento de un proceso de azogue para separar la plata de las gangas; fue nombrado asesor del virrey Toledo en su estancia en México. De regreso a Perú descubrió nuevas minas de mercurio en Huancavelica, lo que le valió el nombramiento de funcionario de la Caja Real de Huamaya y recaudador de arbitrios. No nos extenderemos más en esta época pues la etapa más interesante para nosotros comienza cuando decide bajar su ritmo de trabajo en la minería y retomar su labor como librero y su actividad de promotor cultural.

Esta actividad comenzó en dos frentes: por una parte, trabajó en labores pedagógicas en varios colegios jesuitas y, por otra, comenzó a trabajar para el impresor Antonio Ricardo atendiendo la impresión de textos líricos y profanos en pliegos sueltos, ya que hasta 1596 estuvo prohibido imprimir este tipo de libros. Este trabajo le permitió entrar en contacto con los poetas más importantes del país así como encargar libros a la península. Tal y como afirma Estuardo Núñez, su mérito esencial «reside en haber animado la naciente actividad intelectual del reino peruano y orientado el gusto de los nuevos escritores» ${ }^{8}$ hacia la literatura italiana, se entiende.

Ya en su vejez pidió una pensión de gracia y, ante la lentitud de la burocracia, decidió viajar a España en 1589 para realizar sus peticiones en persona, y con sus ahorros, publicar sus tres traducciones en 1591: Los sonetos y canciones del poeta Francisco Petrarcha; Los Luisiadas de Luis de Camoens; y De reyno y de la institución del que ha de reynar de Francesco Patrizzi.

Sobre su viaje a la península tampoco se ponen de acuerdo los especialistas. Unos afirman que no solo viajó a España, sino que murió en la península alrededor de 1595. Alicia de Colombí ${ }^{9}$ explica que en «diciembre de 1589 llegó a Lisboa tras 44 años de ausencia, en 1591 publicó sus tres libros (...) en Madrid y murió después del 17 de julio de 1593 y antes de 1596»; otros, como Menéndez Pelayo, Antonio Prieto y Justo García Morales insisten en que envió las obras a la península tras lo cual «se hizo presbítero y fue a morir de canónigo en la catedral de México» y que publicó

${ }^{7}$ García Morales, Justo, op. cit., p. 70.

${ }^{8}$ NÚÑEZ, Estuardo, op. cit., p. 134.

${ }^{9}$ De Colombí, Alicia, op. cit., p. 34. Según Estuardo NúŃEZ, op. cit., p. 143, del 17 de julio de 1593 es la real cédula expedida desde El Escorial que concedía a Garcés el principio de una renta sobre la producción de azogue en todo el reino del Perú, por lo que en esa fecha aún vivía. 
«los tres impresos en el mismo año, 1591, porque el autor sin duda los mandó simultáneamente a España» ${ }^{10}$.

En todo caso, lo que está claro es que la traducción se realizó en Perú, pues no es posible que tradujese tres obras de tal envergadura en apenas un año de estancia en Madrid. Además, Estuardo Núñez apunta la idea de que su labor como ayudante de un impresor, junto a su afán como librero, le permitieron difundir en pliegos sueltos los poemas que iba traduciendo. Esta hipótesis explicaría el hecho de que Cervantes hubiera conocido de él lo suficiente como para dedicarle unos elogiosos versos en el Canto de Calíope publicado en 1585, seis años antes de que Garcés viajara a España. ¿De qué otro modo sino?

Dice Cervantes:

De un Enrique Garcés, que al piruano
reino enriquece, pues con dulce rima,
con sutil, ingeniosa y fácil mano,
a la más ardua empresa en él dio cima,
pues en dulce español al gran toscano
nuevo lenguaje a dado y nueva estima,
¿Quién será tal que la mayor le quite,
aunque el mesmo Petrarca resucite? ${ }^{11}$

El propio Garcés, en el primero de los sonetos que dedica a Felipe II en los preliminares de la traducción de la obra de Petrarca, afirma que tuvo su trabajo detenido demasiado tiempo, seguramente porque alternó su labor como traductor y librero con su dedicación a la minería. Todo esto podría reafirmar la hipótesis de una previa circulación de los poemas en pliegos sueltos.

Esta breve aproximación a la figura de Enrique Garcés nos muestra a un hombre comprometido con las letras, que constituye una figura central para el desarrollo de la literatura latinoamericana del último cuarto del siglo XVI y principios del siglo XVII por su labor de traductor y difusor. Nos consta su estrecha relación con los poetas más relevantes del momento, quienes leyeron sin duda sus traducciones de poemas sueltos. Hasta qué punto éstos constituyeron un grupo literario más o menos homogéneo en torno a ideas literarias comunes es un hecho que está por estudiar; sobre todo en relación con la influencia de la literatura italiana, y en especial de Petrarca, línea que permanece abierta para futuras profundizaciones.

En todo caso, hay datos que nos hacen pensar que algo de esto pudo haber. Como ya hemos apuntado anteriormente, Estuardo Núñez explica que, a partir de 1570, Garcés comenzó a difundir sus traducciones en pliegos

10 MENÉNDEZ PELAYO, Marcelino, «Historia de la poesía hispanoamericana», Obras completas de Menéndez Pelayo, Madrid, Librería General de Victoriano Suárez, 1913, Vol. II, p. 199.

11 Cervantes, Miguel de, Canto de Calíope, en La Galatea, Madrid, Cátedra, 1995, p. 578, estrofa 75 . 
sueltos. Esta fecha hemos de relacionarla con el comienzo de su trabajo con el impresor Antonio Ricardo, lo cual indicaría, además, que sus poemas pudieron leerse antes de ser reunidos en un volumen, y que se difundieron durante un largo periodo de tiempo. De hecho, según Nuñez, además de las noticias sobre la traducción de Garcés

también llegaron a España noticias de otros rimadores peruleros, amigos y afines a los gustos renacentistas que compartían comunes aficiones literarias y el gusto italianizante de cultivar sonetos y canciones ${ }^{12}$.

A pesar de que Garcés no tiene más producción propia que los sonetos que incluye en los preliminares y su famosa Canción al Pirú al final del libro - composiciones que tendremos ocasión de analizar más adelante-, esos textos son interesantes, sobre todo en relación con los poetas que se reúnen y que son los que más tarde - alrededor de 1608- acabarían por formar la que se conoce como Academia Atlántica. Esta academia funcionaba ya al menos desde 1580, y fue uno de los centros de mayor difusión del italianismo en Iberoamérica. En ese círculo intelectual, y alrededor de los impresos sueltos de Garcés, trabajaron algunos de los personajes más importantes de la literatura del momento y se compusieron algunas de las obras cumbre de la literatura latinoamericana de la segunda mitad del siglo XVI ${ }^{13}$. Así, Diego de Hojeda y Pedro de Oña; Miguel Cabello Balboa y su Miscelánea Antártica (1586); Diego Dávalos y Figueroa y su Miscelánea Austral (1602) - muy bien estudiada por Alicia de Colombíi ${ }^{14}$; Diego Mexía y el Parnaso Antártico (1608) o Juan de Miramontes y Zuázola y sus Armas Antárticas (1615); sin olvidar una recopilación de poemas sueltos a modo de cancionero llamado Flores de baria poesía que, según Alicia de Colombí, es el «más fehaciente documento del petrarquismo americano, único sin duda en el Virreinato de Perú» ${ }^{15}$.

Todos estos poetas se acercaron a la literatura italiana y, en particular a Petrarca, e intentaron imitar sus modelos, gracias, al menos en parte, a la

12 NÚÑEZ, Estuardo, op. cit., p. 135. Entre los 'rimadores peruleros' a los que alude Nuñez, encontramos, por ejemplo, a Sancho de Ribera, de quien el propio Cervantes dice en el Canto de Calíope, op. cit., p. 578, estrofa 75: «con sus divinos versos alegrando,/ Venga y veréis por suma deste cuento, / su heroico brío y discreción mirando,/ que es Sancho de Ribera, en toda parte Febo primero y sin segundo Marte». Del mismo modo, en la estrofa 76, encontramos a otro de los poetas incluidos en la obra de Garcés, Rodrigo Fernández de Pineda: «Un Rodrigo Fernández de Pineda, /cuya vena inmortal, cuya excelentel y rara habilidad, gran parte hereda (...) Pues de tal gloria goza en Occidente (...) cual la merecen hoy su ingenio y arte».

13 Sobre este círculo de intelectuales véase el texto de Luis Monguió titulado Sobre un escritor elogiado por Cervantes: los versos del perulero Henrique Garcés y sus amigos, Berkeley y Los Angeles, University of California Press, 1960.

14 DE COLOMBÍ, Alicia, op.cit., passim.

15 Íbid., p. 11. 
labor de Garcés. Todos ellos han sido muy bien estudiados por los especialistas en literatura latinoamericana, sin embargo, muchas preguntas quedan aún sin respuesta, y no sólo con respecto a la vida del portugués: queda por estudiar el trabajo de Garcés independientemente del estudio de la obra de Petrarca, así como el desarrollo de esas influencias petrarquistas en los círculos peruanos y latinoamericanos del momento.

Queremos aquí profundizar en la primera de las líneas: la traducción de Enrique Garcés. Como hemos indicado anteriormente, la obra fue publicada en Madrid en 1591 por el impresor Guillermo Droy ${ }^{16}$, quien mantuvo una imprenta entre 1578 y 1599 en la que imprimió pocas pero importantes obras. De Garcés imprimió, además de Los sonetos y canciones, la traducción de Los luisiadas de Camoens; y de su taller salieron también obras como el Arte de retórica de Rodrigo de Espinosa en 1578 o el Quilatador de plata, oro y piedras preciosas de Juan de Arfe Villafañe en $1598{ }^{17}$.

La traducción se inicia con las debidas autorizaciones y privilegios, tras lo que comienza una parte preliminar en la que Garcés incluye varios sonetos a diversos personajes. Los tres primeros poemas van dirigidos a Felipe II; el más interesante es el primero ya que los otros dos son sólo alabanzas al monarca. En el soneto «A Philippo II deste nombre, monarcha primero de las Españas e Indias Oriental y Occidental» Garcés apunta algunas cuestiones que pueden reforzar la hipótesis que hemos expuesto anteriormente en torno a las fases de composición de su texto. Garcés confiesa al monarca:

Siendo este mi trabajo detenido

algunos años más que Horacio manda

(vs.1-2)

La redacción del texto le llevó a Garcés un largo período de tiempo, siempre en Perú, aunque fuese publicado después en España.

Tras esta introducción comienza una de las partes más interesantes del texto: los sonetos que intercambia el traductor con otros poetas limeños y bolivianos del momento, como Pedro Sarmiento de Gamboa, conocido navegante pontevedrés que escribió una Historia de los Incas, a finales del siglo XVI, horrorizado ante la situación de los indígenas del lugar; el dominico fray Jerónimo Valenzuela, también poeta; Rodrigo Fernández de Pineda, poeta peruano; el licenciado Francisco Emanuel y el Licenciado Villarroel $\mathrm{y}$, por último, otro dominico, fray José Miguel de Montalvo.

Estos sonetos tienen la función, en realidad, de justificar la traducción, ya que todos los poetas muestran estar ansiosos por tener una traducción de los versos de Petrarca y alaban a Garcés por haberla emprendido y, tam-

\footnotetext{
16 Ver nota 2.

17 MARSÁ, María, La imprenta en los Siglos de Oro, Madrid, Laberinto, 2001, p. 132. Esta autora sitúa erróneamente la traducción de Petrarca en el año 1598.
} 
bién, por haber elegido el castellano y no el portugués, que era su lengua materna. Estos poemas se articulan como una especie de juego académico en el que unos sonetos responden a otros, como era tradicional en la lírica precedente.

La temática era de esperar. Garcés pide a los diversos poetas que revisen su texto y lo corrijan, a lo que aquellos responden argumentando que no se atreverían a intervenir en un trabajo de tan buena factura. Entre las referencias a lo esperada que era la traducción encontramos, por ejemplo, la de Pedro Sarmiento de Gamboa, quien en el poema Eiusdem ad eodem afirma:

tanto da más Garcés que dio Petrarca; que el tal a sola Italia se reparte: el nuestro, al uno y al otro hemisfero

(vv. 11-13)

En el mismo tono escribe el propio Garcés a Rodrigo Fernández:

$$
\begin{aligned}
& \text { quien viese, si quiera algo desbastada } \\
& \text { por esa mano en todo diligente } \\
& \text { una rima tan perdida de la gente } \\
& \text { del único Petrarca trasladado }
\end{aligned}
$$

(vv. 1-4)

o en el poema «El traductor»:

$$
\begin{aligned}
& \text { Seguid pluma el trabajo començado } \\
& \text { no paréis ni tengáis de cosa espanto } \\
& \text { mirad que's exercicio bueno y santo } \\
& \text { y aún es quiçá de muchos deseado. }
\end{aligned}
$$

(vv.1-4)

Después, el título de otro de los sonetos A ciertos amigos que querían ver esta traducción reafirma la idea que Garcés quería dejar bien clara: que la comunidad intelectual peruana esperaba la traducción y la predilección que dichos poetas sentían por la cultura italiana y, sobre todo, por Petrarca.

Son además curiosos los poemas que se intercambian el propio Garcés y el dominico fray Miguel de Montalvo. En primer lugar porque contienen un artificio muy peculiar: están escritos en varias lenguas a la vez. De este modo, el primero de ellos, de Miguel de Montalvo a Garcés, se titula Trilingüe del mismo presentado y contiene el primer verso en latín, el segundo en italiano y el tercero en castellano, secuencia que se repite hasta el final:

Henrice, gloria gentis lusitanae, à cui le muse cosi amato hanno, que puedes compararte al Mantuano, ille latinem loquens, et tu hispanem. Per te habbiam le cithare thoscane En elegante verso castellano (vv.1-8) 
La respuesta de Garcés no es menos ingeniosa pues a estas tres lenguas añade el portugués y escribe:

\author{
Ninhum mortal ja canse nem se engane \\ en procurar el Pindo tan lexano, \\ poi ch'in voi sacro Mont'alvo soprano \\ tripudiant Latie et Thuscae et musae Hispanae.
}

(vv.1-4)

Además del singular uso de las lenguas, otra peculiaridad llama la atención, un artificio retórico que imita sin duda a Petrarca: el juego de palabras con el nombre del amigo: Montalvo/Mont'alvo (vv.3) al modo del Laura/ l'aura petrarquista.

En la canción Al traductor y a su trabajo, Garcés se dirige, no a sus amigos, sino a su propia obra y ofrece en ella algunos elementos importantes. A parte de varias referencias al largo período de composición de su obra, justifica el hecho de haber realizado la traducción al castellano y no al portugués:

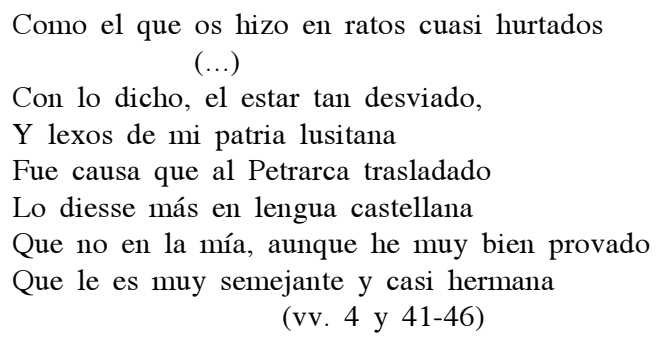

Y en el primer soneto dedicado a Felipe II justificaba la elección de la lengua de destino de su traducción diciendo «y que en ser portugués no desconfíe / pues me assegura el hierro de tu marca» (vv.13-14).

Garcés justifica también en este poema la omisión de la canción XXIX del Canzoniere, Verdi panni, sanguigni, oscuri o persi, por la dificultad del texto petrarquesco:

\footnotetext{
mas ay!, que un verde panni, todo entero me tiene avergonçado, y muy corrido, por no poder supplir tan chica mengua con la riqueza de una, o de otra lengua. Es el Petrarcha allí tan intrincado que no pude passar aquel barranco, ansí me resumí que era acertado dexarle libremente el campo franco, para otro puede ser que esté guardado; bien es que se le quede el papel blanco.
} 
Prueve pues a supplir algún buen genio

la falta de mi pobre y rudo ingenio ${ }^{18}$

(vv. 53-64)

En su lugar el lector halla el incipit de la canción y el resto del folio en blanco, dejando constancia gráfica de su incapacidad para superar el escollo del genio de Petrarca. Falta, sin embargo, una declaración similar en el caso de otros cinco sonetos también omitidos por el traductor ${ }^{19}$.

El caso es que no parece ni un caso de censura ni un fallo de impresión pues el orden de los folios, las palabras reclamo etc. son correctos en los lugares en que esos poemas faltan, salvo por el hecho de que esos poemas no están; además, es también correcta la numeración de las composiciones, no hay saltos, ni tampoco restos de posibles censuras. Todo parece indicar que, o no los tradujo por voluntad propia, o esos poemas no estaban en el original italiano que Garcés utilizó para su traducción, o que, habiendo sido censurados, no fueron incluidos en la selección definitiva.

Al final de la traducción, el autor coloca algunos poemas de poetas italianos coetáneos de Petrarca y amigos personales de éste como Stramazzo de Perugia, Geri de Arezzo, Giovanni Dondi y Jacobo Colonna. Todos en respuesta a poemas del Canzoniere dedicados a ellos por el poeta italiano. La cuestión más importante que queda por resolver con respecto a estos poemas es la procedencia y el porqué de su aparición. Algunos especialistas han opinado que «la lengua traducida no es el toscano sino el latín, y los autores vertidos lo son de poca importancia, limitada nombradía y relativo valor literario» ${ }^{20}$. Esta afirmación es en realidad producto de las incógnitas por resolver que hemos planteado.

Lo cierto es que en realidad esos poemas no estaban originalmente en latín sino en italiano y, además, creemos que la razón por la que están ahí tiene que ver con el posible original en el que basó su traducción Garcés, y no con el hecho de que fueran buenos o renombrados poetas. Para empezar, todos fueron amigos de Petrarca, y todos cuentan con poemas dedicados por el escritor italiano en el Canzoniere.

De hecho, Juan de Dondi no es otro que el paduano Giovanni Dondi

${ }^{18}$ GARCÉS, Enrique, Los sonetos y canciones del poeta Francisco Petrarcha que traduzía Henrique Garcés de lengua thoscana en castellana, Madrid, Guillermo Droy, 1591, «El traductor», vv. 40-48, 53-60.

${ }^{19}$ Los cinco sonetos que faltan son: De l'empia Babilonia ond'è fuggita (CIV); L'avara Babilonia à colmo il sacco (CXXXVII); Fiamma del ciel sule tue treccie piova (CXXXVI); Fontana di dolore, albergo d'ira (CXXXVIII); Mia benigna fortuna e'l viver lieto (CCCXXXII). Falta, además, un fragmento que va del v. 27 al v. 79 de la canción LXXIII, Poi che per mio destino; en este caso, sin embargo, la omisión se debe a la falta del folio 39 que se produjo bien por un fallo de impresión bien por una mutilación posterior a la publicación.

${ }^{20}$ NÚŃEZ, Estuardo, op. cit., p. 142. 
dell'Orologio ${ }^{21}$, amigo y médico de Petrarca, cuya relación vemos en algunas de las cartas que componen los libros XII, XIII y XVI de las Senili ${ }^{22}$. Dondi fue precisamente quien dio testimonio de la muerte de Petrarca en una carta dirigida a otro médico, Giovanni dell'Aquila, al día siguiente del fallecimiento del poeta, en la que dice «una malattia che lo ha colpito di frequente, e che ora lo ha soprafatto» ${ }^{23}$ Es más, aparece incluso en el Testamentum donde Petrarca dice:

Per questa considerazione ho rinviato all'ultimo quello che avrebbe dovuto essere il primo, il maestro Giovanni dell' Orologio, fisico. A lui lascio cinquanta ducati d'oro per comprarsi un piccolo anello d'oro da portare al dito per mio ricordo ${ }^{24}$.

Petrarca lo nombra, además, en el soneto CCXLIV del Canzoniere, aquel que comienza Il mal mi preme, e mi spaventa il peggio que, según los críticos ${ }^{25}$, Petrarca escribió en respuesta a otro de Giovanni Dondi que comenzaba Io non so ben s'io vedo quel ch'io veggio ${ }^{26}$ que es justamente el soneto que Garcés traduce en su obra.

Lo mismo sucede con los otros poemas. El de Stramazzo da Perugia, La santa fama de la qual son privi quasi i moderni, halla respuesta, según reza el propio título de Garcés, en el soneto XX de Petrarca, Se l'onorata fronde che prescrive.

En cuanto a Jacobo Colonna, se trata del amigo Giacomo Colonna quien escribe el soneto Se le parti del corpo mio destrutte, al que contestaba supuestamente Petrarca con el soneto CCCXXII, Mai non vedranno le mie luci asciutte.

Por último, el poema de Geri de Arezzo, Messer Francesco, chi d'amor sospira, recibe respuesta de Petrarca en el soneto CLXXIX, Geri, quando talor meco s'adira.

Esta erudición, no sólo de los poemas de Petrarca sino también de aquellos que sus amigos dirigieron al poeta italiano, y a los que éste respondió, no puede ser obra sola del portugués; cabría pues preguntarnos: ¿por qué esos poemas y esos poetas en concreto?

21 BARBERI SQUAROTTI, Giorgio (dir.), Storia della civiltà letteraria, Torino, UTET, 1996, vol. I, p. 817.

22 PetrarCA, Francesco, Epistole a. c. di Ugo Dotti, UTET, 1978, Senili, XII, I e 2, XIII, 14 y $15, \mathrm{XVI}, 3$.

${ }^{23}$ Ibid., p. 821.

24 PETRARCA, Francesco, «Testamentum», Opere latine di Francesco Petrarca, a.c. di Anonietta Bufano, Torino, UTET, 1978, p. 1352-1353.

25 Veáse por ejemplo: PetrarcA, Francesco, Rime, Trionfi, e poesie latine., a. c. di Ferdinando Neri, G. Martellotti, E. Bianchi e Natalino Sapegno, en La letteratura italiana, storia e testi, Milán, Riccardi editori, 1951.

${ }^{26}$ Los versos originales italianos han sido extraídos de la edición del Canzoniere de 1553 realizada por G. A. GESUALDO titulada Il Petrarcha colla spositione di misser G. A. Gesualdo, Venecia, G. A. Niccolini da Sabbio e fratelli. Nosotros hemos consultado un ejemplar de la reedición de 1574 . 
Esta pregunta junto con las características del texto nos hicieron plantearnos a partir de qué ejemplar italiano había trabajado Garcés su traducción. Para intentar aproximar una idea, hemos cotejado algunas de las ediciones más conocidas de la primera mitad del siglo XVI ${ }^{27}$, es decir, la que incluye el comentario de Alessandro Vellutello, la de Ludovico Castelvetro, la de G.A. Gesualdo, la de Giorgio Angelieri, o la de Bernardino Stagnino.

La única edición sobre la que podemos efectuar una hipótesis es la de G.A. Gesualdo, cuya primera edición es de $1553^{28}$. Esta obra contiene, antes del texto petrarquesco propiamente dicho, cuatro poemas de otros tantos poetas italianos que, en efecto, son los mismos cuatro sonetos italianos que traduce Garcés en su texto, incluyendo el comentario sobre el poema de Petrarca con el que se relaciona cada uno de ellos. Así Gesualdo incluye:

Stramazzo da Perugia al Petrarca risposta a le consonanze del Petrarca $s e$ «l'honorata fronde, che prescrive» título al que sigue el soneto en italiano La santa fiamma de la qual son privo.

Geri di Gianfigliacci al Petrarca. Risposta del Petrarca a le consonanze «Geri, quando talhor meco s'adira», y el soneto Messer Francesco ch d'amor sospira.

Giovanni de Dondi a Misser Francesco Petrarca. Risposta alle consonanze «Il mal mi preme et mi spaventa il peggio» y el soneto Io non so ben s'io vedo quel ch'io veggio.

Y, por último, Iacomo Colonna a Messer Francesco Petrarca. Risposta $a$ «Mai non vedranno le mie luci asciutte» con el soneto Se le parti del corpo mio destrutte.

Es cierto que buena parte de las diferencias de orden que hay en la traducción de Garcés no están en el texto de Gesualdo; sin embargo, sí hay otras como, por ejemplo, todas las de la segunda parte del Canzoniere, incluido el punto en que termina la primera parte y comienza la segunda - con el soneto Ohime il bel viso, ohime il bel sguardo- así como los tres poemas con los que finaliza la obra.

En la edición de Gesualdo aparecen, sin embargo, todos los poemas que no tradujo Garcés. Dado que este libro fue sometido a censura es probable que el mismo Garcés no los tradujera, o bien que fueran suprimidos por el censor antes de la publicación de la obra.

Garcés traduce también un texto de otro poeta italiano, Paolo Pansa, para su hija Ana Garcés, que era monja.

Para acabar con las composiciones que Garcés añade a su traducción, nos falta comentar la Canción al Pirú. Se trata de un texto que, como el

${ }^{27}$ Si Garcés estaba ya en América a mediados de siglo, puede que se llevara la edición consigo, en todo caso, sabemos que a partir de 1570 ya había comenzado su labor, por lo que la edición italiana debe ser anterior.

${ }^{28}$ Ver nota 26. 
propio autor declara, fue escrito A imitación de «Italia mia, ben che'l parlar sia indarno». Lo cierto es que más que una imitación es una versión ${ }^{29}$.

Garcés traduce únicamente la primera y la última estrofa del poema de Petrarca, y las utiliza como pretexto para hablar de los problemas económicos de la zona y, sobre todo, de los problemas mineros y de impuestos sobre la plata que tanto le preocupaban, dado sus intereses en la minería que ya hemos comentado. De hecho, la pieza está considerada como la primera reivindicación, la primera muestra de amor por la patria de América escrita por un peninsular en el siglo XVI.

Para terminar queremos mencionar las dos ediciones modernas de esta traducción. La primera, realizada por Justo García Morales en 1963 y la segunda, llevada a cabo por Antonio Prieto en 1985. Ambos autores intervienen en el texto de manera importante, alterándolo sin advertencia. En concreto la primera de ellas, la de Justo García Morales, restablece el orden de los poemas adecuándolo al orden establecido por Gianfranco Contini basado en el códice vaticano 3195; además, el autor puntualiza en su introducción que ha rellenado los vacíos dejados por Garcés traduciendo por su cuenta los poemas que faltaban e intercalándolos, pero no dice cuáles son esos poemas, por lo que es imposible saber, sin previa collatio, qué textos fueron traducidos por Garcés y cuáles por García Morales.

La segunda edición de la obra de Garcés va aún más lejos, pues además de basarse en la de Justo García Morales en cuanto al orden y a la traducción de los poemas que faltan, elimina los poemas laudatorios, tanto los de Garcés como los de los poetas latinoamericanos e italianos de los que hemos hablado. Por tanto, presenta únicamente los poemas de Petrarca sin tener en cuenta la obra en su conjunto, olvidando el valor de la obra de Garcés para el desarrollo de la literatura española en Hispanoamérica.

Esta actitud respecto del texto de Garcés no es nueva, ya Menéndez Pelayo calificó los versos de Garcés como

incorrectos y desabridos, mal acentuados muchas veces, llenos de italianismos y lusitanismos, como quien calca servilmente en vez de traducir de un modo literal, y no se hace cargo de la diferencia de las lenguas ${ }^{30}$

Sin embargo, traducir es siempre traicionar, produce belle infedeli como decía Gianfranco Folena ${ }^{31}$. Se olvidan quienes juzgan así la traducción de Garcés de que es la única traducción completa ${ }^{32}$ de las rimas de Petrarca

${ }^{29}$ Consúltese el apéndice I con la edición de ambos textos a fronte.

30 MENÉNDEZ PELAYO, Marcelino, op. cit., p.199.

31 FolenA, Gianfranco, Vogarizzare e tradurre, Torino, Einaudi, 1991, p. 74.

${ }^{32}$ La traducción de Samul Usque aunque anterior - la publicó Nicolao Bevilacqua en Venecia en 1567- como es sabido se limita a la primera parte de las rimas, la conocida como In vita di madonna Laura. Se tituló Los sonetos, canciones, mandriales y sextinas del gran poeta Francisco Petrarca, traducidos del toscano por... Parte Primera. Cfr. MANE- 
que hemos tenido desde el siglo XVI hasta la segunda mitad del siglo XX, y que el verdadero valor del texto reside en que abrió paso a la influencia de la literatura italiana, y sobre todo de Petrarca, que será tan importante a partir de ese momento.

$\mathrm{Su}$ valor como texto literario ha sido despreciado por muchos que han alterado incluso su fisonomía original, porque han olvidado que los textos literarios de nuestra historia tienen valor por sí mismos, y que la forma original no es un capricho sino que integra el texto y nos muestra también la forma en que se conoció y difundió la obra en su tiempo.

Tal y como Guillermo Lohmann Villena afirma, «la versión de Garcés no es hoy pura arqueología literaria, sino que hay en ella algunas perlas que sólo necesitan de la mano amorosa que las rescate de la ganga sin valor estético» ${ }^{33}$. Esperamos que este modesto estudio contribuya a que eso suceda y se restituya a la obra de Garcés la importancia que realmente tiene como iniciadora y fomentadora del petrarquismo en el ambiente literario americano.

\section{APÉNDICE I}

\section{CXXVIII $^{34}$}

Italia mia, benchéll parlar sia indarno

a le piaghe mortali

che nel bel corpo tuo sì spesse veggio,

piacemi almen che' miei sospir sian quali
Canción al Pirü ${ }^{35}$

Aunque mi hablar, Pirú, venga a ser vano a daños tan notables,

como en tu cuero y tan continuos sientes, querría fuesen tanto lamentables

RO SOROlla, M. ${ }^{a}$ Pilar, «La primera traducción de las Rime de Petrarca en lengua castellana: los sonetos, canciones, mandriales y sextinas del gran poeta y orador Francisco Petrarca, de Salomón Usque», Homenaje a Antonio Vilanova, ed. A. Sotelo Vázquez, M. Cristina Carbonell, Barcelona, Universidad, 1989, vol I, pp. 377-391; hay además una tesis doctoral inédita que se defendió en 2001 en la Universidad Autónoma de Barcelona sobre la figura de Salomón Usque y su traducción del Canzionere [CANALS, Jordi: Salomón Usque. Traductor del Canzionare de Petrarca]. Después de la de Garcés, en 1595, Francisco Trenado de Ayllón realizó otra traducción incompleta «que no llegó a publicarse, quizá víctima de cierta saturación en aquellos años de finales del XVI o de la buena acogida que tuvo la traducción de Garcés, y que incluso ha pasado desapercibida para parte de la crítica» que se conserva manuscrita en la British Library -Ms. EG. 2062- con fecha en Madrid, 20 de septiembre de 1595. Sobre esta traducción habló la profesora Eva Muñoz Raya en una conferencia titulada «El petrarquismo en España: entre la traducción didáctica y la imitación» sostenida en el marco del Seminari Internacional d'Estudis cap al VIIé Centenari del naixement. Francesco Petrarca, celebrado en Valencia en diciembre de 2002 .

${ }^{33}$ LOHMANN Villena, Guillermo, «Henrique Garcés, descubridor del mercurio en el Perú, poeta y arbitrista», Anuario de Estudios Americanos, V, 1948, pp. 439-482.

${ }^{34}$ Petrarca, Francesco, Cancionero, Jacobo Cortines (ed. y trad.), Madrid, Cátedra, 1984, vol. I, págs. 468-475.

35 GaRCía MORALES, Justo, op.cit., pp. 406-410. 
spera 'l Tevero, et l'Arno

$e$ 'l Po, dove doglioso et grave or seggio.

Rettor del cielo, io cheggio

che la pietà che ti condusse in terra

ti volga al tuo dilecto almo paese;

vedi, Segnor cortese,

di che lievi cagion che crudel guerra;

$e$ i cor, che 'ndure et serra

Marte superbo et fero

apri tu, Padre, e 'ntenerisci, et snoda;

ivi fa' che ' $l$ tuo vero,

qual io mi sia, per la mia lingua s'oda.

Voi, cui Fortuna à posto in mano il feeno de le belle contrade,

di che nulla pietà par che vi stringa,

che fan qui tante pellegrine spade?

Perché ' $l$ verde terreno

del barbarico sangue si depinga?

Vano error vi lusinga;

poco vedete, et parvi veder molto,

ché ' $n$ cor venale amor cercate o fede.

Qual più gente possede

colui è più da' suoi nemici avolto.

$O$ diluvio raccolto

di che deserti strani

per inondar i nostri dolci campi!

Se da le proprie mani

questo n'avene, or chi fia che se ne scampi?

Ben provide Natura al nostro stato, quando de l'Alpi schermo

pose fra noi et la tedesca rabbia;

ma ' $l$ desir cieco, e 'ncontra ' $l$ suo ben fermo,

s'è poi tanto ingegnato,

ch'al corpo sano à procurato scabbia.

Or dentro ad una gabbia

fiere selvagge et mansüete gregge

s'annidan sì che il miglior geme:

et ̀̀ questo del seme,

per più dolor, del popol senza legge,

al qual, come si legge,

Mario aperse sì 'l fianco,

che memoria de l'opra ancho non langue,

quando, assetato et stanco,

non più bevve del fiume acqua che sangue.

Cesare taccio, che per ogni piaggia

fece l'erbe sanguigne

di lor vene, ove ' $l$ nostro ferro mise. los versos de mi mano

que a compasión moviesen todas gentes.

A ti vuelvo mis mientes,

rector del cielo, y pido no consientas

que este rincón del todo se consuma;

que no es tan chica summa

la que de tus ovejas apacientas

en él, si bien las cuentas,

que no sean hato entero.

Suplícote, Señor, que no se diga

que olvidas este apero,

y mira tu pasión a qué te obliga.

¡Oh vos, a quien las hondas dio y cayados deste nuevos rebaños

el rabadán mayor con larga mano!

¿Cómo no dais remedio a tantos daños?

¿No veis que, si atajados

no son, que irán cundiendo todo el llano

que estaba a partes sano?

Si creéis que eso que hacéis es acertado, mirad qué muestra os da de lo contrario el mal tan ordinario

que cada día va más entablado,

sin que haya aprovechado

haberse antes fundido,

que entra por mil caminos y mil puertos.

$\mathrm{Y}$ pues que esto es sabido,

dad orden como cesen tantos tuertos.

Bien proveído había al pobre estado aquel pastor que puso

el septo contra tanta desventura.

Mas, ¡ay!, que siempre el bien es intercluso; $\mathrm{y}$, en fin, ello ha parado

en desterrar de aquí la plata pura,

$\mathrm{y}$ agora una mixtura

quieren que tome el pobre jornalero

qu'es plomo, estaño y cobre sin estima

¡Mirad si hay por qué gima

el malaventurado! Que el dinero

que le paga el minero,

al traer el tributo,

le dice el oficial muy rasamente

y con mando absoluto:

-No es paga. Y para el pobre es competente.

No trato lo de atrás, que ya la tierra está bien sosegada,

aunque a gran costa fue de sus entrañas. 
Or par, non so per che stelle maligne, che ' $l$ cielo in odio n'aggia: vostra mercé, cui tanto si commise. Vostre voglie divise guastan del mondo la più bella parte. Qual colpa, qual giudicio, o qual destino fastidire il vicino povero, et le fortune afflicte et sparte perseguire, $e$ ' $n$ disparte cercar gente, et gradire

che sparge ' $l$ sangue et venda l'alma a prezzo?

Io parlo per ver dire, non per odio d'altrui né per disprezzo.

Né v'accorgete anchor per tante prove del bavarico inganno ch'alzando il dito colla morte scherza? Peggio è lo strazio, al mio parer, che 'l danno.

$M a$ ' $l$ vostro sangue piove

più largamente, ch'altr'ira vi sferza.

Da la matina a terza

di voi pensate, et vederete come

tien caro altrui chi tien sé così vile.

Latin sangue gentile,

sgombra da te queste dannose some;

non far idolo un nome

vano senza soggecto;

ché 'l furor de lassù, gente ritrosa,

vinverne d'intellecto,

peccato è nostro, et non natural cosa.

Non è questo 'l terren ch'i' tocchai pria? Non è questo il mio nido

ove nudrito fui sì dolcemente?

Non è questa la patria in ch'io mi fido,

madre benigna et pia,

che copre l'un et l'altro mio parente?

Per Dio, questo la mente

talor vi mova, et con pietà guardate

le lagrime del popol doloroso,

che sol da voi riposo

dopo Dio spera; et pur che voi mostriate

segno alcun di pietate,

vertù contra furore

prenderà l'arme, et fìa 'l combatter corto, ché l'antiquo valore

ne l'italici cor non è anchor morto.

Signor, mirate come'l tempo vola, et sí come la vita
Una visita nuevamente hallada

es la que le da guerra

agora más cruel, pues las cabañas

les vacía por mil mañas;

y no falta quien diga que consiste

en ella todo el bien ¡Oh qué buen medio!

Publícase remedio,

y quitan la comida al pobre triste,

$\mathrm{y}$ al otro lo que viste.

$\mathrm{Y}$ si hay quien pagar quiera

lo que comió, lo cuenta a menos precio

allá en cierta manera,

y da por plata en cobre y plomo el precio.

$\mathrm{Ni}$ mil ensayos han aprovechado contra tan grande engaño,

que a la tierra acarrea mal tan fuerte,

cuyo tormento no es menor qu'el daño

que, si habéis procurado

querer comer con paga desta suerte, es una pura muerte,

que no hay pasarla al sol, ni ya que asombre, que a la candela mucho más paresce lo por donde envilesce, qu'es cobre disfrazado en otro nombre. ¿Pues qué ha de hacer el hombre con tal desgañamiento como es esta mixtura cautelosa de gentes perdimiento, nueva invención y no natural cosa?

No creo que ha dexado en vuestro oído de penetrar el llanto qu'en derredor de aqueste valle suena con tanta confusión, horror y espanto; que, si no es sin sentido,

no hay tigre que no sienta en verlo pena: que, en la casa llena de niños, si el pan falta es gran tormento, y mucho más si han sido regalados.

¡Ay pobres desdichados

los hijos deste valle! Pues descuento

a vuestro descontento ninguno lo es pasado: pan, pan, pan es la falta más urgente, que esotro es ya olvidado.

Haya en esto siquiera un diligente.

¿No es esta aquella tierra que solía con un celo no frío 
fugge, et la morte n'è sovra le spalle. Voi siete or qui; pensate a la partita; ché l'alma ignuda e sola conven ch'arrive a quel dubbioso calle. Al passar questa valle, piàcciavi porre giù l'odio et lo sdegno, vènti contrari a la vita serena;

et quel che ' $n$ altrui pena

tempo si spende, in qualche acto più degno o di mano o d'ingegno, in qualche bella lode, in qualche honesto studio si converta. Così qua giù si gode, et la strada del ciel si trova aperta.

Canzone, io t'ammonisco

che tua ragion cortesemente dica, perché fra gente altera ir ti convene, et le voglie son piene già de l'usanza pessima et antica, del ver sempre nemica.

Proverai tua ventura

fra magnanimi pochi a chi ' $l$ ben piace; di' lor: «Chi m'assicura?

I'vo gridando: «Pace, pace, pace»». mil pobres socorrer muy francamente? ¿No es esta la provincia del gran brío, madre benigna y pía, que con su haber honrado ha tanta gente? Suplico os humilmente, que piedad y justicia en vos no muera ¡Mirad el triste pueblo doloroso que, de vos, el reposo después de Dios con gran derecho espera! Si hacéis reales fuera irá del todo el daño, y el reino andará luego en gran concierto; que aquel vigor de antaño

aun en Pirú no está del todo muerto.

Mirad que el tiempo vuela y que la vida tan corta es como incierta,

y que del paso horrendo nadie escapa y que es bien que nuestra alma ande despierta

y prompta a la partida, que no cata a señor, ni a rey ni a papa, ni al que no tiene capa.

Pues, para poder ir más descansados y no perder la vía más serena, -que el peso da gran penaserá muy conveniente ir muy aliviados de todos los cuidados que nos presenta el suelo, y en obras buenas todo se convierta, que nos se gana el cielo si desde acá no va la senda abierta.

Ten en cuenta, canción mía, que vayas con humilde reverencia, que has de ir a razonar con gente altiva, $\mathrm{y}, \sin$ mostrarte esquiva, presenta a donde fueres tu consciencia, ni temas de pendencia.

Ve, prueba tu ventura, sin que des muestra alguna de alterada. ¿Y a ti quién te asegura?:

El que la paz dexó tan encargada.

\section{BIBLIOGRAFÍA}

BARBERI SQUAROTTI, Giorgio. (dir.), Storia della civiltà letteraria, Torino, UTET, 1996. BELLINI, GIUSEPPE, Historia de la literatura latinoamericana, Madrid, Castalia, 1990. BeRMúdeZ-GAllegos, Marta, «Petrarquismo y aculturamiento: «La canción al Piru» de Henrique Garcés», Romance Languages Annual, 1, 1989, pp. 384-389.

CERVAntes, Miguel de, La Galatea, Francisco López Estrada y M. Teresa López GarcíaBerdoy (eds.), Madrid, Cátedra, 1995. 
DE Colombí, Alicia, Petrarquismo peruano: Diego Dávalos y Figueroa y la poesía de la Miscelánea Austral, Londres, Tamesis Books Limited, 1985.

Dizionario biografico degli italiani, Roma, Istituto della Enciclopedia Italiana, 1999.

FOLENA, Gianfranco, Vogarizzare e tradurre, Torino, Einaudi, 1991.

GARCÉS, Enrique, Los sonetos y canciones del poeta Francisco Petrarcha que traduzia Henrique Garcés de lengua thoscana en castellana, Madrid, Guillermo Droy, 1591.

GARCía MORAlES, Justo, Rimas en vida y muerte de Laura; Triunfos, Madrid, Aguilar, 1963.

GARRIBBA, A, «La prima traduzione completa dei Rerum vulgarium fragmenta in spagnolo: Los sonetos y canciones del Petrarcha, que traduzía Henrique Garcés de lengua thoscana en castellana (Madrid, 1591), Artifara, n. 3 (luglio-dicembre 2003), http:// www.artifara.com/rivista3/testi/petrarca.htm

-, «Il petrarchismo in America Latina nel Cinquecento: il caso di Enrique Garcés», Il confronto letterario, 40, 2003, pp. 247-261.

—, «Aspectos léxicos de la traducción del «Canzoniere» por Enrique Garcés (1591)» Cuadernos de filología italiana, 12, 2005, pp. 115-132.

Gesualdo, Giovanni Andrea, Il Petrarcha colla spositione di misser G.A. Gesualdo, Venecia, Niccolini da Sabbio, 1574

LOHMAN VILlENA, Guillermo, «Enrique Garcés, descubridor del mercurio en el Perú, poeta y arbitrista», Anuario de estudios americanos, V, 1948, pp. 439-482.

MADRIGAL, Luis Ignacio, Historia de la literatura Hispanoamericana, Tomo II, Época Colonial, Madrid, Cátedra, 1982.

MANERo SOROLla, M. a Pilar, «La primera traducción de las Rime de Petrarca en lengua castellana: Los sonetos, canciones, mandriales y sextinas del gran poeta y orador Francisco Petrarca de Salomon Vsque (Venecia 1567)», en Homenaje al Prof. Antonio Vilanova, ed. A. Sotelo Vázquez, M. Cristina Carbonell, Barcelona, Universidad, 1989, Vol. I, pp. 377-391.

- Introducción al estudio del petrarquismo en España, Barcelona, PPU, 1987.

MARSÁ, María, La imprenta en los Siglos de Oro, Madrid, Laberinto, 2001.

MENÉNDEZ PELAYO, Marcelino, «Historia de la poesía hispanoamericana», Obras completas de Menéndez Pelayo, Tomo II, Madrid, Librería General de Victoriano Suárez, 1913.

MONGUIÓ, Luis, Sobre un escritor elogiado por Cervantes: los versos del perulero Henrique Garcés y sus amigos, Berkeley y Los Angeles, University of California Press, 1960.

MUÑOZ RAYA, Eva, «El petrarquismo en España: entre la traducción didáctica y la imitación», conferencia sostenida en el marco del Seminari Internacional d'Estudis cap al VIIé Centenari del naixement. Francesco Petrarca, celebrado en Valencia en diciembre de 2002.

NUÑEZ, Estuardo, «Henrique Garcés, múltiple hombre del Renacimiento», La tradición clásica en el Perú virreinal, Teodoro Hampe (Comp.), Lima, Sociedad Peruana de Estudios Clásicos: Universidad Nacional Mayor de San Marcos, 1999, pp. 129-144.

PETRARCA, Francesco, Cancionero, Jacobo Cortines (ed. y trad.), Madrid, Cátedra, 1984.

-, Rime, Trionfi, e poesie latine, a. c. di Ferdinando Neri, G. Martellotti, E. Bianchi e Natalino Sapegno, La letteratura italiana, storia e testi, Milán, Riccardi Ricciardi editori, 1951 .

—, «Testamentum», Opere latine di Francesco Petrarca, a. c. di Antonietta Bufano, Torino, UTET, 1975, pp. 1348-1351.

—, Epistole, a. c. di Ugo Dotti, Torino, UTET, 1978.

PRIETO, Antonio, Francesco Petrarca. Cancionero, Barcelona, Planeta, 1985. 